\title{
Spectroscopic characterization of different samples of humic acids
}

Sokolova I. ${ }^{1}$, Kudlatskaya A. ${ }^{1}$, Selyanina S. ${ }^{2}$, Naumova L. ${ }^{1}$, Tchaikovskaya O. ${ }^{1}$

${ }^{1}$ National Research Tomsk State University, Tomsk, Russia, sokolova@phys.tsu.ru

${ }^{2}$ Federal Scientific Research Centrum for Complex Learning of the Arctic, Arkhangelsk, Russia

doi: 10.36291/HIT.2019.sokolova.047

Humic substances are the products of extremely complex chemical and microbial processes of the decomposition and secondary synthesis of plant and animal residues in soil and water. Humic substances (including humic and fulvic acids) are naturally photoactive components, which are widely present in environment. Humic substances representing the main fraction of organic matter receive increased attention because of their reactivity as light absorbers. Depending on their origin and structure, humic substances have a remarkable ability to absorb light and transfer this energy to other substrates and in some cases strongly affect photolysis of xenobiotics.

The absorption and fluorescence spectra of different samples of humic acids (HAs) are investigated. The samples of HAs were prepared from peat of Arkhangelsk Region. The comparison of this HAs with the samples of humic acids obtained from Aldrich Chemical Co is carried out.

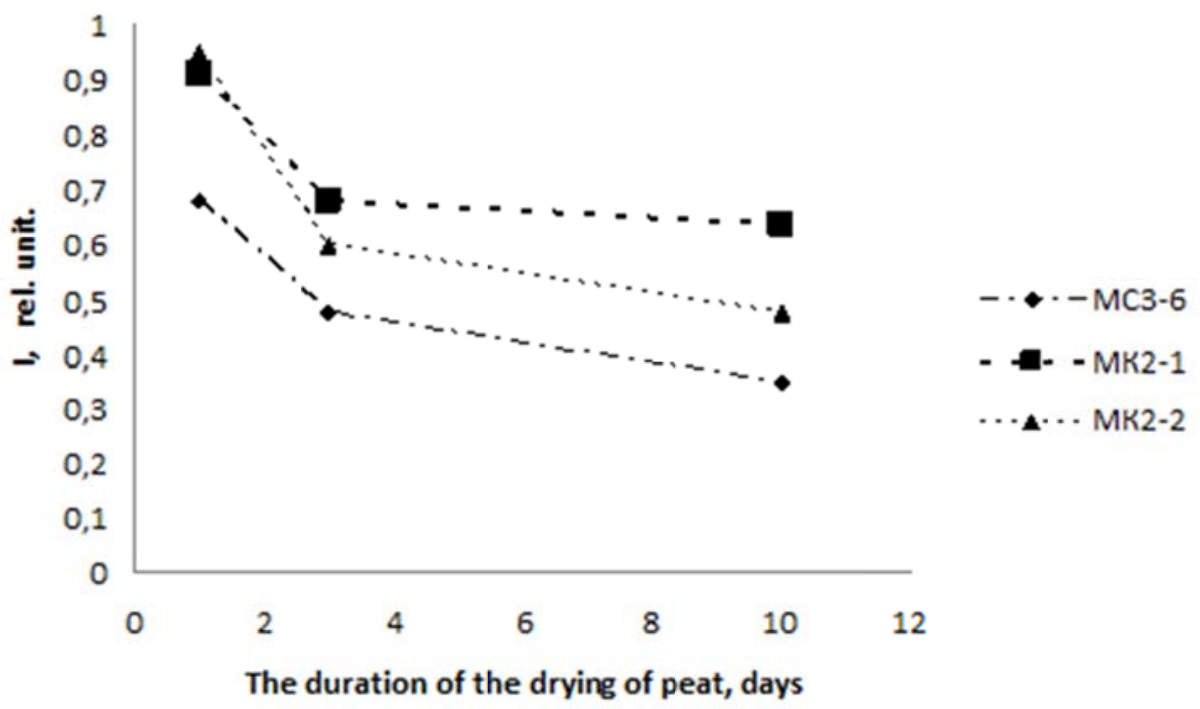

Figure 1. Dependence of the optical density of the absorption spectra of various HAs samples on the drying time.

The longer is the duration of drying of the samples, the lower the optical density of the absorption spectra. In the process of humification and with the duration of peat drying, the fluorescence intensity of the HAs increases. The dependence of the fluorescence spectra of the investigated samples on the fluorescence excitation wavelength was found.

The effect of UV radiation from excilamps on the spectroscopic properties of humic acids has been investigated.

An important point to emphasize is the significance and prospects of optical and photochemical methods in determining the characteristics and functions of humic acids as extremely sensible and informative methods.

Acknowledgements. This work is supported by the Tomsk State University competitiveness improvement program and Russian Foundation for Basic Research (Project № 18-05-60151). 